\title{
REVISTA DE REVISTAS
}

La selección de artículos que ofrecemos a continuación ha sido realizada a partir de las revistas de ciencias sociales correspondientes al año 1981 de que se dispone en las siguientes bibliotecas de la Universidad Autónoma de Barcelona, a 23 de diciembre de 1981.

Biblioteca de la Facultad de Ciencias Económicas

American Journal de Sociology

American Sociological Review

L'Avenç

Berkeley Journal of Sociology

The British Journal of Sociology

Cahiers Internationals de Sociologie

Ciudad y Territorio

Consumation

Critica Marxista

Critigues de l'Economie Politique

Current Sociology

Government and Opposition

The Insurgent Sociologist

International Journal of Contemporary Sociology

The Journal of Peasant Studies

Journal for the Scientific Study of Religion

The New Hungarian Quarterly

New Left Review

Papeles de Economía Española

La Pensée

Population

Population Bulletin

Problemi del Socialismo

Cuadernos de Realidades Sociales 
«Papers»: Revista de Sociologia

Quaderni. Rassegna Sindacale

Recerques

Revista de Estudios Agro-Sociales

Revista Internacional de Sociología

Revista Internacional del Trabajo

Revue Française de Sociologie

Social Compass

The Sociological Review

Sociología del Trabajo

Sociologie du Travail

Soviet Studies

Theory and Society

Les Temps Modernes

Hemeroteca de la Facultad de Ciencias de la Información

CAU

El Viejo Topo

Biblioteca de la Facultad de Derecbo

Documentación Social

Revista Española de Investigaciones Sociológicas

Revista de Política Comparada

Revista de la Seguridad Social

Biblioteca General (Edificio del Rectorado)

Sistema

Cambio social

Monique Dagnand, «La classe "d'alternative". Réflexion sur les acteurs du changement social dans les sociétés modernes», Sociologie du Travail 4 (1981): 384-405.

Albert Soboul, «Qu'est-ce que la Révolution?», La Pensée 217/218 (1981): 33-45.

Oleg Zinam, «Impact of modernization on attitudes toward violence as means of resolving national and international conflicts», International Journal of Contemporary Sociology 3 y 4 (1981): 200-225. 
Revista de revistas

ECOLOGÍA HUMANA Y DEMOGRÁFICA

Lucien Blanco, «Planification des naissances en Chine», Population 1 (1981): 123-146.

Michéle Brahimi, «Chronique de l'immigration», Population 1 (1981): 147-161.

Karl W. Deutsch, «The crisis of the State», Government and opposition 3 (1981): 331-343.

Charlotte Höhn «Les différences internationales de mortalité infantile: illusion ou réalité»?, Population 4/5 (1981): 791-816.

Alain Lefebvre et Alfred Sauvy, «Influence de l'évolution démographique sur les changes sociales», Population 2 (1981): 251-265.

Alain Monnier, «La reprise récente de la natalité dans quelques pays d'Europe occidentale», Population 4/5 (1981): 897-921.

Mirjama Morokvasik-Muller, «Prendre les risques: femmes inmigrées entre Ia contraception et l'avortement», Les Temps Modernes 418 (1981): 1933-1953.

Guy Standing, «Migration and modes of explotation: social origins of inmobility and mobility», The Journal of Peasant Studies 2 (1981): 173-211.

HISTORIA, TEORÍA Y METODOLOGÍA

Bruno Accarino, «La razionalità in Weber», Critica Marxista 1 (1981): 113-120.

Robert J. Antonio, "Inmanent critique at the core of critical theory: its origins and developments in Hegel, Marx and contemporary thought», The British Journal of Sociology 3 (1981): 330-345.

Erika Apfelbaum, «Origins de la psycologie sociale en France. Développements souterrains et discipline méconnue», Revue Française de Sociologie 3 (1981): 397-407.

Phillip Bossermann, «Georges Gurvitch et les durkheimiens, en France, avant et aprés la seconde guerre mundiale», Cabiers Internationales de Sociologie, enero-junio (1981): 111-126.

Denis Duclos, «Project éthique et positivisme dans la démarche sociologique de Durkheim», Cabiers Internationales de Sociologie, enero-junio (1981), 101-110.

Jean Claude Dutailly, «Peut-on sauver le marxisme»?, Les Temps Modernes 417 (1981): 1764-1791.

R. Eyerman y D. Shipway, «Habermas on work and culture», Theory and Society 4 (1981): 547-578.

Arturo Fittipaldi, «Roberto Longhi e la cultura dei beni culturali», Critica Marxista 1 (1981): 129-148. 
«Papers»: Revista de Sociologia

Robert Fraisse, «Les sciences sociales: utilisation, dépendence, autonomie», Sociologie du Travail 4 (1981) 369-383.

Etienne Géhin, «Rousseau et l'historie naturelle de l'homme social», Revue Française de Sociologie 1 (1981): 15-31.

Roger L. Geiger y René Worms, «L'organicisme et l'organisation de la sociologie», Revue Française de Sociologie 3 (1981): 361-395.

Marita Gilli, «L'Émergence de la pensée matérialiste en Allemagne», La Pensée 219 (1981): 5-17.

Richard Guillàn, «White collar from start to finish: C. Wright Mills», Theory and-Society 1 (1981): 130.

Felipe González Vicen, «Filosofía y revolución en los primeros escritos de Marx», Sistema 40 (1981): 3-34.

Felipe González Vicen, «La crítica de Marx y la escuela histórica», Sistema $43 / 44$ (1981): 3-23.

Richard A. Hilbert, «Toward an improved understanding of role», Theory and Society 2 (1981): $207-226$.

Robert J. Holton, «Marxist theories of social change and the transition from feudalism to capitalism», Theory and Society 6 (1981): 833 . 867.

Edith Kurzweil, «Jacques Lacan: French Freud», Tbeory and Society 3 (1981), 419-438.

Chistofer Lash, «The freudian left and cultural revolution», New Left Review 129 (1981): 23-34.

Carles Lemert y Paul Piccone, «Alvin W. Gouldner: Reflections and Memories», Tbeory and Society 2 (1981): 163-167.

Ian Lubek, «Histoire de psychologies sociales perdues. Le cas de Gabriel Tarde», Revue Française de Sociologie 3 (1981).

Peter Ludes, «Marx's notnon of a classless society», International Journal of Contemporary Sociology 3 y 4 (1980): 1-53.

Virginio Marzocchi «Ernst Bloch: metafísico dell'utopia o filosofo della prassi?», Crítica Marxista 1 (1981): 101-112.

Donald McQuarie, «The pain marxism of C. Wright Mills», Tbe Insurgent Sociologist 3 (1981): 83-94.

Artur Mitzman, «Flaubert and Weber. Post-heroic consciousness in France and Germany», Tbeory and Society 1 (1981): 81-102.

L. Saavedra, «La racionalización en Max Weber», Sistema 42 (1981): 93-117.

Antonine Savoge: «Les continuateurs de Le Play au tournant du siècle», Revue Française de Sociologie 3 (1981): 315-344.

Yvon J. Thiec, «Gustave Le Bon prophète de l'irrationalisme de masse», Revue Française de Sociologie 3 (1981): 409-428.

Alain Touraine, «Une sociologie sans société», Revue Française de Sociologie 1 (1981): 3-13.

Stephen A. Turner y Regis A. Factor, «Objective possibility and adequate 
causation in Weber's methodological writings», The Sociological $R e$ view 1 (1981).

Sergio Vilar, «Fourier y la revolución del placer», El Viejo Topo 53 (1981): 15-19.

Raymond Williams, «Marxism, structuralism and literary analysis», New Left Review 129 (1981): 55-66.

ORganizaCión SOCIAL, SISTEMa SOCIAL, ESTRUCTURA SOCIAL

György Aczél, «Answers-questions on Hungarian society», The New Hungarian Quarterly 82 (1981): 122-142.

Nickey Britten, «Models of intergenerational class mobility: findings from the national survey of health and development», The British Journal of Sociology 2 (1981): 224.238.

Michael C. Burrage y David Corry, "Ocupational status in 14th-17th Century London», American Sociological Review 4 (1981): 375-393.

Héctor Díaz Polanco, «Etnia, clase y cuestión nacional», El Viejo Topo 59 (1981): 16-21.

Marie-Thérèse Duflos-Priot, «L'apparience individuelle et la representation de la réalite humaine et des clases sociales», Cabiers Internationales de Sociologie, enero-junio (1981): 63-84.

Mark Elchardus, "Class, structuration and achievements», The Sociological Review 3 (1981): 413-444.

Cristóbal González Román, «Ordres, status i classes a l'antiguitat grecorromana», L'Avenç 40 (1981): 65-70.

Nan Lin, W. M. Ense1, J. C. Vaughn, «Social resources and strenght of ties», American Sociological Review 4 (1981): 393-405.

Chris Middleton, «Peasants, patriarchy ond the feudal mode of production in England. A marxist appraisal», The Sociological Review 1 (1981): 105-154.

Pedro Molas i Ribalta, «L'orgull social de la petita burgesia a l'Antic Règim: la "cascada del menyspreu"», L'Avenç 38 (1981): 52-55.

Glenn Morgan, "Class theory and the structural locations of black workers», The Insurgent Sociologist 3 (1981): 21-34.

Otthein Rammsted, «Masses. From an idealistic to a materialistic point of view? Aspects on marxian theory of the class», International Journal of Contemporary Sociology 3/4 (1981): 55-79.

Laurence Ratier-Coutrot, «Changer de place ou changer de classe: mobilité professionelle et trajet social», Sociologie du Travail 4 (1981): 406-429.

H. A. Roberts y K. Sevee, «Trends in ocupational mobility: Canada and US», American Sociological Review 4 (1981): 406-421.

Carlota Solé, «Integración versus catalanización de los emigrantes», Sistema 43/44 (1981): 171-197.

Andrés Sorel, «Comida y lucha de clases», El Viejo Topo 53 (1981): 44-45. 
Jadniga Staniszkis, «The evolution of forms of working class pretest in Poland: Sociological reflections on the Gdansk-Sczecin Cesc», Soviet Studies 2 (1981): 201-231.

José Félix Tezanos, «Identificación de clase y conciencia obrera entre los trabajadores industriales», Sistema 43/44 (1981): 87-123.

Pierre Vilar, «Réflexions sur les fondements des structures nationals», La Pensée 217/218 (1981): 46-64.

\section{Números monográficos}

Dossier: "Marginats, fronterers, rebels $i$ oprimits: xuetes, "1laneros", gitanos i pehl», L'Avenç 42 (1981): 17-36.

«Marginación social en España», Documentación Social 44 (1981).

\section{SOCIOLOGía DEL CONOCIMIENTO Y DE LA CULTURA}

John Alt, «Authority, reason, and the civilizing process», Theory and Society 3 (1981): 387-405.

Roberta Ascarelli, "Comunicazione di massa e movimento operario. Le origine», Critica Marxista 1 (1981): 66-99.

Pierre Boiteau, «De la pluralité des modes de connaissance», La Pensée 220 (1981): 6-16.

Jesús Contreras y Joan Prat, «L'Estudi de la cultura popular», L'Avenç 44 (1981): 63-65.

Robert D. Cumming, "Giving back words: things, money, persons», Social Research 2 (1981): 227-259.

Vittoria Franco: «USA: la cultura del narcisismo», Critica Marxista 1 (1981): 121-128.

Janine Goetschy, «Les théories du pouvoir», Sociologie du Travail 4 (1981): 447-467.

Ralph Heydels, «L'ideologie. Critique d'une notion», Cabiers Internationales de Sociologie, enero-junio (1981): 157-168.

Charles C. Lemert, "Literary politics and the Champ of French sociology, Theory and Society 5 (1981): 645-669.

François Mariet, «L'imperialisme culturel, la pédagogie et les media: le charme discret de l'imperialisme», La Pensée 221/222 (1981): 101-113.

H. C. F. Mansilla, «La función instrumental de la ética y de la ideología en sistemas socialistas», Sistema 42 (1981): 129-136.

Ignacio Sotelo, «El concepto sociológico de crisis», Sistema 40 (1981): 25-37.

Lilly Weisbrod, «From labour Zionism to new Zionism: ideological change in Israel», Theory and Society 6 (1981): 777-803. 
Revista de revistas

SOCIOLOGÍA ECONÓMICA Y DEL DESARROLLO

Julián Briz Escribano, «La ganadería española en la década de los ochenta: consideraciones socioeconómicas», Revista de Estudios Agro-Sociales 115 (1981): 131-136.

G. A. Cohen, «Freedom, justice and capitalism», New Left Review 126 (1981): 3-16.

Nora Hamilton, «State autonomy and dependent capitalism in Latin América», The British Journal of Sociology 3 (1981): 305-329.

Caglar Keyder, «Proto-industrialization and the periphery: a marxist perspective», The Insurgent Sociologist 3 (1981): 51-57.

Johan Galtung, «El nuevo orden internacional y el enfoque de las necesidades básicas», Revista de Política Comparada 3 (1980/1981): 11-36.

Robert Thomas, "The social organization of the industrial agriculture», The Insurgent Sociologist 3 (1981): 5-20.

\section{SOCIOLOGÍA DE LA EDUCACIÓN}

Ken Browne, «Schooling, capitalism and the mental/manual division of labour», The Sociological Review 3 (1981): 445-473.

Alexandre Cirici, «Els estudiants i la cultura a l'època de la República», L'Avenç 38 (1981): 12-13.

Gerd Dury, «Factores socioemocionales condicionantes de la conducta de aprendizaje en el adulto», Cuadernos de Realidades Sociales 18/19 (1981): 181-200.

Roberto Fernández Díaz y Elena Sierco Barón, «Educació professional i desenvolupament econòmic: les escoles de la Junta de Comerç», L'Avenç 37 (1981): 58-61.

Francisco J. Laporta, «La libertad de enseñanza, Constitución y Estatuto de Centros Docentes», Sistema 40 (1981): 39-54.

Carlos Lerena, «Autodidaxia y nueva cultura», Sistema $43 / 44$ (1981): 57-73.

José Ángel López Herrerías, «Consecuencias actuales y líneas de corrección en el sistema escolar del isomorfismo entre el estamentalismo (feudal) y el inicio de las Universidades», Cuadernos de Realidades Sociales 18/19 (1981): 245-250.

Federico Mayor Zaragoza, «La Universidad en el mundo actual», Revista de Política Comparada 4 (1981): 11-19.

Alain Mingat, "Aptitudes et classes sociales, accès et succès dans l'enseignement supérior», Population 2 (1981): 337-358.

James Murphy, "Class inequality in education: two justifications, one evaluation but not hard evidence», The British Journal of Sociology 2 (1981): 182-201. 
«Papers»: Revista de Sociologia

Ángel I., Pérez Gómez, «Ideología y ciencia en la escuela», Cuadernos de Realidades Sociales 18/19 (1981): 201-213.

SOCIOLOGía DE LA FAMILIA Y GRUPOS DE EDAD

A. Cheslin y P. B. Walters, «US men's and Women's sex role attitudes», American Sociological Review 4 (1981): 453-460.

László Cseh-Szombathy, «The family, its members and society», The New Hungarian Quarterly 82 (1981): 54-62.

Patrick Festy y Jean-Louis Rallu, «Constitution et reconstitution des familles françaises. Etat civil et recensement de 1968 a 1975», Population 1 (1981): 63-91.

Alain Paraut, «Les personnes agées en France et leurs conditions d'habitat», Population 3 (1981): 577-619.

Louis Roussel, «Le remariage des divorcés», Population 4/5 (1981): 765 790.

David M. Smith, «New movements in the sociology of youth: a critique», The British Journal of Sociology 2 (1981): 239-251.

Paloma Villegas, «El feminismo devastador», El Viejo Topo 56/57 (1981).

Hélène Ivert-Jalu, «L'histoire du divorce en Russie soviétique. Ses raports avec le politique familiale et les réalités sociales», Population 1 (1981): 41-61.

Virgilio Zapatero, «Comentarios al proyecto de ley de divorcio», Sistema 41 (1981): 3-39.

Gary Wray McDonogh, «Societat anònima i parentiu a Barcelona» (1829. 1953), L'Avenç 43 (1981): 63-67.

\section{Números monográficos}

Dossier: «El divorci: aproximació a una història», L'Avenç 38 (1981): $25-42$.

SOCIOLOGÍA JURÍDICA Y CRIMINAL

Santiago Mir Puig, «Problemática de la pena y seguridad ciudadana», Sistema 43/44 (1981): 75-85.

Marino Barbero, «La pena de muerte en la Constitución», Sistema 42 (1981): 31-56.

Ennesto Garzón Valdés, «Acerca de la desobediencia civil», Sistema 42 (1981): 79-92. 
Mercedes Alcover Ibáñez y Luis Vila López, «El hospital como organización: un nuevo enfoque», Revista de Seguridad Social 10 (1981): 133-163.

M. Ángeles Durán, «Estructura social y enfermedad en España», Revista de Seguridad Social 10 (1981): 195-209.

F. J. Yuste Grijalba, «Ensayos sobre higiene mental. De Freud a Fromm», Sistema 40 (1981): 119-125.

\section{Números monográficos}

«La población española ante las drogas», Documentación Social 42 (1981). «Salud y Sociedad», Documentación Social 43 (1981).

\section{SOCIOLOGÍA POLÍTICA}

Odile Dhavernas, «Feminisme et institutions: une enquête a Quèbec», Les Temps Modernes 418 (1981): 1903-1932.

Andree Michael, «Mouvements Féministes en Occident et projet de société», Les Temps Modernes 414 (1981): 1270-1284.

José Luis Abellán, «Orígenes del pensamiento económico y las ideas socialistas en España», Sistema 40 (1981), 101-117.

Roberto Bergalli, «Proyectos hegemónicos y estado autoritario en Argentina», Sistema 42 (1981): 17.29.

Francisco J. Bobillo, «Intelectuales y política», Revista de Política Comparada 4 (1981): 117-148.

David Coates, "Labourism and the transition to socialism», New Left Review 129 (1981): 3-22.

S. N. Eisenstadt, «Cultural traditions and political dynamics: structuring of collectivities and accountability of rulers», The British Journal of Sociology 2 (1981): 155-181.

Luis García San Miguel, «Las ideologías políticas en la España actual», Sistema 40 (1981): 55-77.

Francis Ginsbourger, «Japon: voyage au pays du consensus social», Les Temps Modernes 415 (1981): 1319-1351.

Alfonso Guerra, «Situación política tras el golpe de Estado», Sistema 42 (1981): 3-15.

Jeffrey Herf, «Reactionary modernism. Some ideological origins of the primacy of politics in the Third Reich", Theory and Society 6 (1981): 805-832.

Tomás Ibáñez, «La inevitabilidad del poder político y la irresistible ascensión del poder coercitivo», El Viejo Topo 60 (1981): 28-33. 
Eric M. Leifer, "Competing of political mobilization: the role of ethnic ties», American Journal of Sociology 1 (1981).

Rafael López Pintor, «El estado de la opinión pública española y la transición a la democracia», Revista Española de Investigaciones Sociológicas 13 (1981): 7-47.

Rodolfo Martín Villa, «Análisis sociológico de la transición política», Revista de Politica Comparada 4 (1981): 21-35.

Diana Pinto, «Sociology, politics and society in postwar Italy, 1950-1980», Theory and Society 5 (1981): 671-705.

Manuel J. Risques, «La insurrecció de Barcelona pel novembre de 1849. La seva dinàmica social», Recerques 10 (1980): 93-112.

\section{SOCIOLOGÍA DE LA RELIGIÓN}

Hart M. Nelsen, «Life afterlife: towards congruency of belief across generations», Journal for the Scientific Study of Religion 2 (1981): 10-118.

John J. Nelson y Harry, «Norms of verbalization and the decision making process in religious organizations», Journal for the Scientific Study of Religion 2 (1981): 173-180.

David A. Nock, «The historical procees and the reformulation of religious typologies: The case if the Anglican communion», The Sociological Review 3 (1981): 521-541.

Robin Theobald, «The politization of religious movement: British adventisen under the impact West Indian inmigration», The British Journal of Sociology 2 (1981): 202-223.

\section{Números monográficos}

Karel Dobelaere, «Secularization: a multidimensional concept», Current Sociology 2 (1981). Se trata el concepto de secularización como proceso de laicización y de cambio religioso, así como en su multidimensionalidad. Hace un recuento de las teorías de la secularización. Ofrece una amplia bibliografía comentada de Sociología de la Religión.

\section{SOCIOLOGÍA DEL TRABAJO Y ORGANIZACIONES}

Rafael Aracil, «Per una història de la societat pagesa tradicional: les formes de treball (XI). El treball tèxtil», L'Avenc 41 (1981): 44-51.

Michael Blanc, «Le prix de la fidelité. Essai de modélisation des stratégies de carrière des militaires», Revue Française de Sociologie 1 (1981): 5-86. 
François Bourguignon, «Participation, emploi et travail domestiques des femmes mariées», Consumation 2 (1981): 75-98.

Sergio Bruero, «Ricerche ilimiti delle riarche sulle politiche dell'impiego», Quaderni Rassegna Sindicale 90/91 (1981): 131-140.

Cristina Calandre, Javier Buscones y Nieves Navarro, «Los impactos de la General Motors», Ciudad y Territorio 2 (1981): 19-39.

Juan J. Castillo y Carlos Prieto, «Una técnica subjetiva de investigación en condiciones de trabajo: las encuestas de satisfacción», Revista Española de Investigaciones Sociológicas 13 (1981): 49-63.

Josep M. Delgado i Ribalta, «Per una història de la societat pagesa tradicional: les formes de treball (VIII). La navegació catalana d'altura: els mariners de comerç lliure», L'Avenç 36 (1981): 52-59.

«Per una història de la societat pagesa tradicional: les formes de treball (IX). La construcció naval catalana: els mestres d'aixa», L'Avenç 38 (1981): 44-50.

Jordi Estivill y Antonio Artiles, «Crisis y sindicatos: nuevas estrategias para nuevos tiempos», Sociología del Trabajo 6 (1981): 7-28.

Jean-Paul Gaudemar, «URSS. Le travail fait systeme», Les Temps Modernes 417 (1981): 1792-1815.

Grupo de Trabajo sobre Problemas del Empleo (GTE), «Población, actividad y ocupación en España», Papeles de Economía Española 6 (1981): 209-235.

W. van Ginnieken, «El desempleo: tendencias, causas, posibilidades de acción», Revista Internacional del Trabajo 3 (1981): 285-303.

Enrique González-Duro, «La neurosis del desempleo», El Viejo Topo 60 (1981): 23-27.

George Kanawaty, «Nuevas formas de organización del trabajo: experiencias sobre el terreno», Revista Internacional del Trabajo 3 (1981).

Bertrand Lemennicier y Louis Lèvy-Garboure, «L'arbritage autarciemarché: une explication du travail fémenin», Consumation 2 (1981): 41-74.

Antonio Lucas Marín, «Una aproximación sociológica al estudio de la satisfacción en el trabajo en la provincia de Santander», Revista española de Investigaciones Sociológicas 13 (1981): 65-100.

Jordi Maluquer de Motes, «Per a una història de la societat pagesa tradicional: les formes de treball (VII). L'explotació del bosc i el transport de la fusta (els raiers)» L'Avenç 34 (1981): 36-43.

Tomás Parra, Teresa Rojo y Luis Sanz «Una aproximación al análisis espacial: mercado de trabajo y territorio», Ciudad y Territorio 1 (1981): 7-15.

Víctor Pérez Díaz, «Los obreros españoles ante el sindicato y la acción colectiva en 1980», Papeles de Economía 61 (1981): 236-265.

Ida Regalia, «Sindicalizzazione e mutamenti del mercato del lavoro», Quaderni Rassegna Sindicale 90/91 (1981): 15-28. 
R. Rosanvallon, «La cuestión del trabajo», El Viejo Topo 60 (1981): 19-23.

L. Sayles, E. Sarin, «Les nouvelles formes d'organization du travail, un engouement?», Sociologie du Travail 4 (1981): 430-446.

J. Scott y R. Mcginnis, «Organizational context and scientific productivity», American Sociological Review 4 (1981): $422-442$.

Carles Sudrià «Per una història de la societat pagesa tradicional: les formes de treball (X). La ramaderia transhumant», L'Avenç 39 (1981): $42-49$.

\section{Números monográficos}

«Segmentation de l'emploi ou division du salariat?», Critiques de l'Economie Politique 15-16 (1981).

Confrontación de los empleos estables y los trabajadores estables con los trabajadores y empleos precarios, como resultado de las actuales transformaciones en el empleo.

«Le travail des femmes», Critiques de l'economie politique 17 (1981). Artículos sobre la salarización del trabajo femenino, las trabajadoras jóvenes y la familia, la división sexual del trabajo y el papel del Estado.

«Empleo y desempleo: I. Perspectivas económicas y sociológicas», Revista de Seguridad Social 11 (1981).

«Empleo y desempleo: I. Perspectivas jurídicas y sociológicas», Revista de Seguridad Social 12 (1981).

«Fábrica y Ciudad», Sociología del Trabajo 5 (1981).

Trata de la separación entre fábrica y ciudad, la destrucción de los barrios industriales céntricos, relación entre capitalismo y cambios en los modos de vida en las zonas rurales... Ofrece además bibliografía comentada y recensiones de libros útiles para una profundización en el tema.

«Crisis económica y relaciones de trabajos», Sociología del Trabajo 6 (1961). Artículos sobre el papel de los sindicatos ante la crisis económica y las repercusiones de la misma en las relaciones laborales. Contiene una bibliografía comentada sobre «Crisis económica y sindicalismo».

SOCIOLOGÍA RURAL Y URBANA

Elisa Badosa, «Els lloguers de cases a la ciutat de Barcelona (1780-1834)», Recerques 10 (1981).

Patrick Clapier y Nicole Tabard, «Transformation de la morphologie sociale des communes et variation des consommations», Consumation 2 (1981): 3-40. 
Antonio Fernández Alba, «El lugat de los arquitectos en la sociedad industrial. El ocaso de una profesión», Cau 70 (1981): 42-44.

Antonio Fernández Alba, «La ciudad del destino final», Cau 71 (1981): 50-54.

Claude S. Fischer, «The public and private worlds of city life», American Sociological Review 3 (1981): 306-316.

José A. Garmendia y Juan del Pino, «Desigualdades campo-ciudad: a propósito de algunos comportamientos diferenciales básicos del desarrollo económico-social de la sociedad española, Sistema 41 (1981): 87-103.

José Miguel Hernández León, «Más leña al fuego de la arquitectura postmoderna. Ideología y lenguaje», Cau 71 (1981): 44-48.

Bernard Ineichen, «The housing decisions of young people», The British Journal of Sociology 2 (1981): 252-258.

Irina Iivezeanu, «Urbanization in low key and linguistic change in Soviet Moldavia», Soviet Studies 3 (1981): 327-351.

Fernando Parra, «Ordenación del territorio: receta para urbanistas y maquillaje ecológico», Ciudad y Territorio 2 (1981): $15-18$.

Ivan Szelenyi, «Urban developement and regional management in Eastern Europe», Theory and Society 2 (1981): 169-205.

Constanza Tobío e Ignacio Duque, «Desarrollo y nivel de ejecución en 1979 de los equipamientos previstos en el planeamiento general y parcial de las ciudades medias españolas», Revista Internacional de Sociología 36 (1981): 539-576.

Julio Vidaurre, «Para un equilibrio urbano», Cau 69 (1981): 44-46. 